\title{
The O-glycosylation mutant osteopontin alters lung cancer cell growth and migration in vitro and in vivo
}

\author{
ARASH MINAI-TEHRANI ${ }^{1}$, SEUNG-HEE CHANG ${ }^{1}$, SEUNG BUM PARK $^{2}$ and MYUNG-HAING CHO ${ }^{1,3-5}$ \\ ${ }^{1}$ Laboratory of Toxicology, College of Veterinary Medicine, Seoul National University; ${ }^{2}$ Department of Chemistry, \\ College of National Science, Seoul National University; ${ }^{3}$ Graduate Group of Tumor Biology, Seoul National University; \\ ${ }^{4}$ Graduate School of Convergence Science and Technology, Seoul National University, Seoul 151-742; \\ ${ }^{5}$ Advanced Institute of Convergence Technology, Seoul National University, Suwon, Gyeonggi 443-270, Republic of Korea
}

Received May 27, 2013; Accepted August 27, 2013

DOI: $10.3892 /$ ijmm.2013.1483

\begin{abstract}
Osteopontin (OPN) is an acidic, glycosylated and phosphorylated protein that plays an essential role in determining the aggressiveness and oncogenic potential of several types of cancer, including lung cancer. The OPN function is highly dependent on post-translational modification (PTM) and regulation of the processes that involve OPN can be mediated through glycosylation. However, the connection between OPN function and its O-glycosylation in lung cancer cells has yet to be investigated. In the present study, this issue was addressed by studying the effects of wild-type (WT) OPN and a triple mutant (TM) of OPN, which was mutated at three O-glycosylation sites in lung cancer cells. It was shown that OPN WT rather than OPN TM induced the OPN-mediated signaling pathway. The OPN WT expression enhanced cap-dependent protein translation, $\mathrm{NF}-\kappa \mathrm{B}$ activity and glucose uptake, whereas a reduction was observed in cells treated with OPN TM. The results clearly demonstrated that unlike OPN WT, OPN TM did not increase lung cancer cell growth and migration both in vitro and in a xenograft mouse model. Thus, results of the present study suggested that targeting OPN by introducing OPN TM may be a good strategy for treating lung cancer.
\end{abstract}

\section{Introduction}

Lung cancer has emerged as the leading cause of cancer-related mortalities, with an increasing incidence worldwide. The long-term survival of lung cancer patients treated with conventional therapies, including surgery, radiation therapy and chemotherapy, remains poor and has changed little in

Correspondence to: Professor Myung-Haing Cho, Laboratory of Toxicology, College of Veterinary Medicine, Seoul National University, 1 Gwanak-Ro, Gwanak-Gu, Seoul 151-742, Republic of Korea

E-mail: mchotox@snu.ac.kr

Key words: osteopontin, O-glycosylation, lung cancer decades. Therefore, a more rational approach to lung cancer therapy is required.

Osteopontin (OPN) is an acidic, glycosylated, phosphorylated and secreted protein that is crucial in determining the aggressiveness and oncogenic potential of several types of cancer, including lung cancer $(1,2)$. It interacts with specific receptors that affect different cascades of the signaling pathway and modulate the expression of several downstream molecules associated with cancer development. Thus, investigators have attempted to inhibit cancer progression by targeting the OPN function $(3,4)$. The OPN function mainly depends on post-translational modification (PTM) and regulation of the processes that involve OPN may be mediated through glycosylation and phosphorylation $(5,6)$. O-glycosylation is considered a highly complex process in which carbohydrate chains are created and extended through the sequential addition of sugars in a Golgi apparatus wherein transferases are located $(7,8)$.

Five O-glycosylation sites have been identified in humans, three of which are also conserved in a mouse OPN (9-11). However, no reports have indicated the importance of the O-glycosylation sites in OPN, the function of OPN and in regulating its downstream signaling pathway.

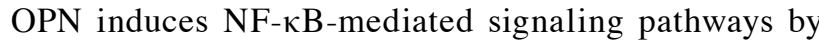
binding to its receptor. $\mathrm{NF}-\kappa \mathrm{B}$ is constitutively active in most tumor cells and its suppression inhibits the growth and migration of tumor cells (12-14). Translation plays an important role in controlling cell growth and it can be regulated via the mammalian target of rapamycin (mTOR) signaling pathway through p70S6K, of which OPN-activated Akt is a critical intermediate. Translation initiation has been shown to be a common downstream target of signal transduction pathways deregulated in several types of cancer, including lung cancer (15-17). However, the mechanism by which OPN regulates mTOR/p70S6K and NF- $\kappa \mathrm{B}$ signaling pathways as well as how inhibition of these pathways may alter cell migration and growth in lung cancer cells remain to be clarified.

In the present study, it was hypothesized that the specific inhibition of OPN downstream signaling by introducing triple mutant (TM) OPN may alter lung cancer cell growth and motility. Therefore, wild-type (WT) OPN and OPN TM were introduced to lung cancer cells and their effects on cap-dependent protein translation, $\mathrm{NF}-\kappa \mathrm{B}$ activity and glucose 
uptake were examined. The effects of OPN WT and TM on lung cancer cell growth and migration both in vitro and in a xenograft mouse model were also investigated.

The present study demonstrated that unlike OPN WT expression, OPN TM expression did not increase cap-dependent protein translation, $\mathrm{NF}-\kappa \mathrm{B}$ activity and glucose uptake. The study results also showed that OPN TM decreased lung cancer cell growth and migration, suggesting that targeting OPN by introducing OPN TM is a promising strategy for lung cancer therapy.

\section{Materials and methods}

Cell culture and materials. H226 and H322 lung cancer cells were purchased from the American Type Culture Collection (CCL-185; Rockville, MD, USA). The cells were grown in RPMI-1640 supplemented with $10 \%$ FBS and $1 \%$ penicillin/streptomycin from Gibco BRL (Grand Island, NY, USA). The mTOR and phospho-mTOR antibodies were obtained from Cell Signaling Technology, Inc. (Beverly, MA, USA). The other indicated antibodies were purchased from Santa Cruz Biotechnology, Inc. (Santa Cruz, CA, USA). LY294002 and monensin were purchased from Calbiochem (San Diego, CA, USA); PDTC and rapamycin from Sigma- Aldrich (St. Louis, MO, USA); and NBD-C6-ceramide from Molecular Probes (Eugene, OR, USA). The bicistronic construct, pcDNA-fLUC-polIRES-rLUC, in which firefly luciferase represents cap-dependent and the renilla luciferase activity represents cap-independent protein translation, was provided by DrGram (Novartis Pharma AG, Basel,Switzerland). The NF- $\kappa$ B luciferase reporter vector was a gift from Dr Nancy Colburn (National Cancer Institute, Frederick, MD, USA). The transfection reagent LT1 was obtained from Mirus Biotechnology (Madison, WI, USA). The Luciferase assay kit and the pRA-SV40 control vector were purchased from Promega (Madison, WI, USA). The lentivirus that contained shRNA targeting p70S6K (shp70S6K), the BLOCK-iT ${ }^{\text {тм }}$ Lentiviral RNAi Expression System (Invitrogen, Grand Island, NY, USA), was generated according to the manufacturer's instructions, after which the virus titer was determined using an HIV-1 p24 ELISA kit from PerkinElmer Life Sciences (Boston, MA, USA).

Plasmid constructs and generation of a stable cell line. The human OPN was generated via reverse transcription-PCR (RT-PCR) using the total RNA. Primers used for RT-PCR were: a forward primer that contained an $\mathrm{XbaI}$ restriction site (5'-GCTCTAGAATGAGAATTGCAGTGATTTG-3') and a reverse primer that contained a $K p n I$ restriction site $\left(5^{\prime}-\mathrm{CGG}\right.$ GGTACCTTAATTGACCTCAGAAGATGC-3'). The PCR product was inserted into the corresponding sites of pcDNA3.1(-) to generate pcDNA3.1(-)/OPN WT. Point mutations on threonine in each glycosylation site (T120A, T124A, T129A, T133A and T138A) of OPN and three point mutations of OPN (T124A, T133A and T138A; OPN TM), as well as five point mutations of OPN in which all five OPN glycosylation sites were converted to alanine $(5 \mu \mathrm{m})$, were generated using pcDNA3.1(-)/OPN WT as a template. The final constructs were confirmed via restriction enzyme analysis and sequencing. To generate a stable cell line, pcDNA3.1(-)/OPN WT or TM was transfected into the $\mathrm{H} 226$ cells using the TransIT ${ }^{\circledR}$-LT1 reagent. The transfected cells were selected with a medium that contained $100 \mathrm{~g} / \mathrm{ml}$ of G418 disulfate salt from SigmaAldrich and the selected cells were maintained in $50 \mathrm{~g} / \mathrm{ml}$ of G418 disulfate salt for future experiments.

$R T$-PCR. Total RNA was isolated using a TRIzol reagent, after which RT-PCR was performed with the One-Step RT-PCR kit (Intron, Seongnam, Korea), according to the manufacturer's instructions. The primers used were: OPN forward, 5'-GCAG AATCTCCTACCCCAC-3' and reverse, 5'-TCGGAATGC TCATTGCTCTC-3'; GALNac-T1 forward, 5'-CTGCCATGG TAGGTGTCCTG-3' and reverse, 5'-TGAGGCTTGGAG CACACTTC-3'; and GAPDH forward, 5'-GAAGGACTCAGA CCACAG-3' and reverse, 5'-CTTCACCACCTTCTTGATG-3'. GAPDH was amplified and used as an internal control. The products were analyzed via electrophoresis on $1 \%$ agarose gels.

Western blot analysis. The total protein concentration in the cell lysates, media and homogenized tumor samples was determined using the Bio-Rad Protein Assay reagent (Bio-Rad, Hercules, CA, USA). Equal amounts of protein were separated via SDS-PAGE and transferred to nitrocellulose membranes. The membranes were blocked in Tris-buffered saline with Tween-20 (TBST) that contained 5\% skim milk. Immunoblotting was performed by overnight incubation with the indicated primary antibodies at $4^{\circ} \mathrm{C}$ and then with HRP-conjugated secondary antibodies for $3 \mathrm{~h}$. The bands were detected using the luminescent image analyzer LAS-3000 (Fujifilm, Tokyo, Japan). The results of the western blot bands analysis were quantified with Multi-gauge v2.02 software (Fujifilm).

Immunohistochemistry (IHC). Fixed tumors were embedded in $10 \%$ neutral phosphate-buffered formalin. The paraffinembedded tissue sections were cut and transferred to plus slides. The sections were deparaffinized in xylene and rehydrated through alcohol gradients. To quench the endogenous peroxidase activity, the sections were incubated in $3 \%$ hydrogen peroxide for $10 \mathrm{~min}$. After being washed in PBS, the sections were incubated in PBS with 3\% bovine serum albumin (BSA) for $1 \mathrm{~h}$ at room temperature to block non-specific binding sites. Corresponding primary antibodies were applied and the sections were incubated overnight at $4^{\circ} \mathrm{C}$. The following day, they were washed and incubated with secondary HRP-conjugated antibodies (1:50; Zymed) for $3 \mathrm{~h}$ at room temperature. After being washed, the sections were incubated with DAB (Vector Laboratories, Burlingame, CA, USA) and counterstained with Mayer's hematoxylin (Dako, Glostrup, Denmark). The slides were then imaged under a light microscope (Carl Zeiss, Gottingen, Germany).

OPN immunoassay. The cells were transfected with OPN WT and TM constructs. OPN levels from a cultured medium were then determined via Quantikine Human OPN Immunoassay from R\&D Systems (Minneapolis, MN, USA). The results were read on a microplate reader from Bio-Rad at $450 \mathrm{~nm}$ OD.

Immunofluorescence assay. The cells were grown and treated on a two-well chamber slide. The slides were washed with PBS and fixed in $4 \%$ paraformaldehyde and methanol, and 
in acetone, respectively. After being blocked with 3\% BSA, the sections were incubated with specific primary antibodies, washed and incubated with fluorescence-conjugated secondary antibodies. Their nuclei were stained with DAPI. The slides were visualized using a fluorescent microscope (Carl Zeiss).

In vivo tumor xenograft studies. Six-week-old nude mice were obtained from Joongang Laboratory Animals, Inc. (Seoul, Korea). The mice were maintained in a pathogen-free animal facility at least one week before use. The nude mice were inoculated subcutaneously with $\mathrm{H} 226$ cells that were non-transfected or stably transfected with OPN WT or TM. Seven mice were used in each group. The volume of the tumor in each mouse was measured with a caliper at regular intervals and calculated as described previously $(18,19)$. At the end of the experiment, the mice were sacrificed and their individual tumors were weighed and collected for subsequent analysis.

Luciferase assay. The treated cells were lysed in a passive lysis buffer from Promega and centrifuged for $10 \mathrm{~min}$ at $4^{\circ} \mathrm{C}$. A supernatant was used for the luminescence dose. The LucR and LucF activities were measured using a Dual Luciferase kit from Promega according to the manufacturer's instructions.

Cy3-labeled glucose uptake. $\mathrm{H} 226$ cells that stably expressed OPN WT and TM were cultured on glass. Then, $6 \mu \mathrm{M}$ of Cy3-labeled glucose (53) was added, and the viable cells were monitored using confocal laser scanning microscopy (CLSM) within $15 \mathrm{~min}$. Fluorescent images of the cells were captured every $60 \mathrm{sec}$.

Wound healing assay. The confluent $\mathrm{H} 226$ cell monolayers were scratched with a pipette tip, with 30 min pre-incubation in the presence of mitomycin. The wound areas were observed using phase contrast microscopy on an inverted microscope. Images of the same areas were captured at regular intervals over the indicated time.

Invasion assay. Invasion assays were performed using BD BioCoat $^{\mathrm{TM}}$ Matrigel $^{\mathrm{TM}}$ Invasion Chambers with $8 \mu \mathrm{m}$ polycarbonated filters from BD Biosciences (San Jose, CA, USA) according to the manufacturer's instructions.

Nuclear extraction. The nucleus and cytosol were separated with the Nuclear Extract kit from Active Motif (Carlsbad, CA, USA). The cells were grown to confluence in a $100-\mathrm{mm}$ tissue culture plate and washed with $5 \mu 1$ ice-cold PBS/phosphatase inhibitors. The collected cells were gently resuspended in a $500 \mu \mathrm{l}$ hypotonic buffer and incubated on ice for $15 \mathrm{~min}$. After their incubation, a $25 \mu$ ldetergent was added in the tube. The cytoplasmic fraction and the nuclear pellet were separated through centrifugation. The nuclear pellet was resuspended with a $50 \mu \mathrm{l}$ complete lysis buffer and incubated for $30 \mathrm{~min}$ on ice on a rocking platform at $150 \mathrm{rpm}$. Following centrifugation, the supernatant containing nuclear proteins was collected for further analysis.

Statistical analysis. Statistical analyses were performed with a Student's t-test for experiments on two groups using the Graphpad Software (San Diego, CA, USA).

\section{Results}

Expression of OPN WT and TM in the H226 cells. To characterize the connection between OPN O-glycosylation sites and their function, a series of point mutations in O-glycosylation sites were introduced into the OPN gene and the cells were co-transfected with OPN WT or O-glycosylation mutant forms of OPN and $\mathrm{NF}-\kappa \mathrm{B}$ luciferase or bicistronic luciferase construct. The results clearly showed that the introduction of selective mutation on three O-glycosylation sites of OPN reduced the $\mathrm{NF}-\kappa \mathrm{B}$ activity and the cap-dependent protein translation more potently than other O-glycosylation mutant forms of OPN (Fig. 1A and B). Based on the results, OPN TM was chosen for subsequent experiments.

The mRNA levels of OPN following the transfection of the H226 cells with OPN WT and TM were then examined. The results clearly showed that the mRNA level of OPN increased in the OPN WT/TM-transfected cells (Fig. 1C). The immunofluorescence assay also showed the overexpression of OPN in Golgi in the cells transfected with OPN WT and TM, unlike in the control (Fig. 1D). The mRNA level of GalNAc-T1 in the $\mathrm{H} 226$ cells was also determined. Unlike OPN WT, OPN TM did not increase the mRNA level of GalNAc-T1 (Fig. 1E). The western blot analysis and ELISA revealed that unlike OPN TM, OPN WT increased the expression levels of OPN in conditioned media (Fig. 1F and G). It was also demonstrated that treatment of OPN WT/TM-transfected cells with PMSF, a broad-spectrum protease inhibitor, increased the OPN expression levels (Fig. 1G).

OPN WT, but not OPN TM, induced the OPN-dependent signaling pathway. To evaluate the mechanisms of OPN regulation and the role of OPN substrates in OPN-dependent biologic responses in cell migration and growth, western blotting, immunofluorescence and invasion and wound migration assays were carried out in cells transfected with OPN WT/TM. The results clearly showed that unlike OPN TM, OPN WT increased Akt, p-Akt (Thr308/Ser473), IKK, mTOR, p-mTOR and Glut-1, as determined by the western blot and densitometric analyses (Fig. 2A). Unlike OPN WT, OPN TM did not increase the expression level of p70S6K, p-p70S6K and uPA (Fig. 2B). The immunofluorescence assay reconfirmed this pattern (Fig. 2C). Furthermore, the wound healing and invasion assays demonstrated that OPN WT rather than OPN TM increased cell migration and the number of invaded cells (Fig. 3).

OPN TM did not increase the glucose uptake, cap-dependent protein translation and $N F-\kappa B$ activity. Stable $\mathrm{H} 226$ cell lines that expressed OPN WT and TM were generated to further explain the effects of OPN WT and TM on glucose uptake and the OPN-dependent signaling pathway. The results clearly showed that the glucose uptake increased in the cells that stably expressed OPN WT, but did not increase in the cells that stably expressed OPN TM (Fig. 4). The altered expression levels of the key proteins involved in the NF- $\mathrm{B}$ signaling pathway when the stable cells were transfected with Akt were investigated. The expression of Akt increased the expression levels of phosphorylated forms of Akt at Thr308 and NF- $\kappa \mathrm{B}$ p65 in cytosol, whereas the NF- $\kappa \mathrm{B}$ p65 expression in the nucleus decreased. GAPDH and PARP were used as controls 
A

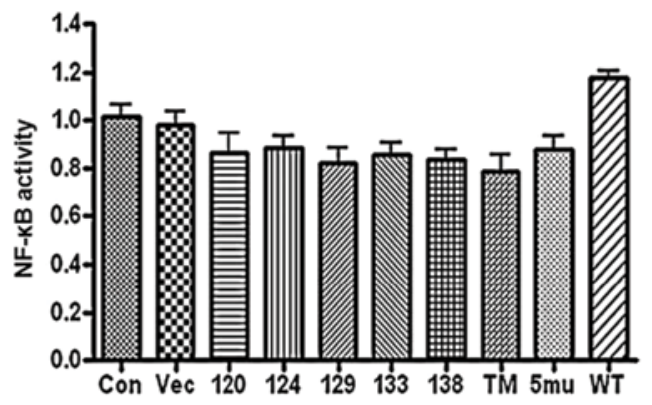

B

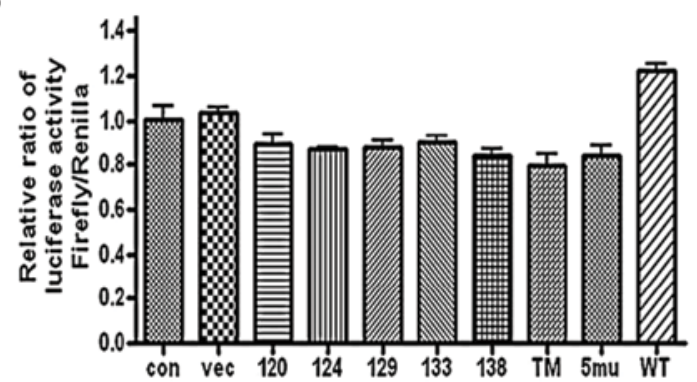

C

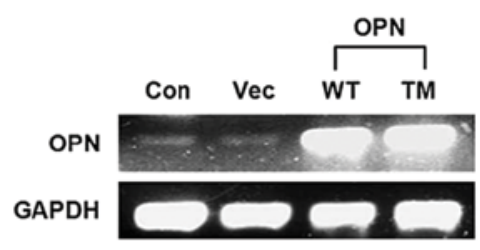

D

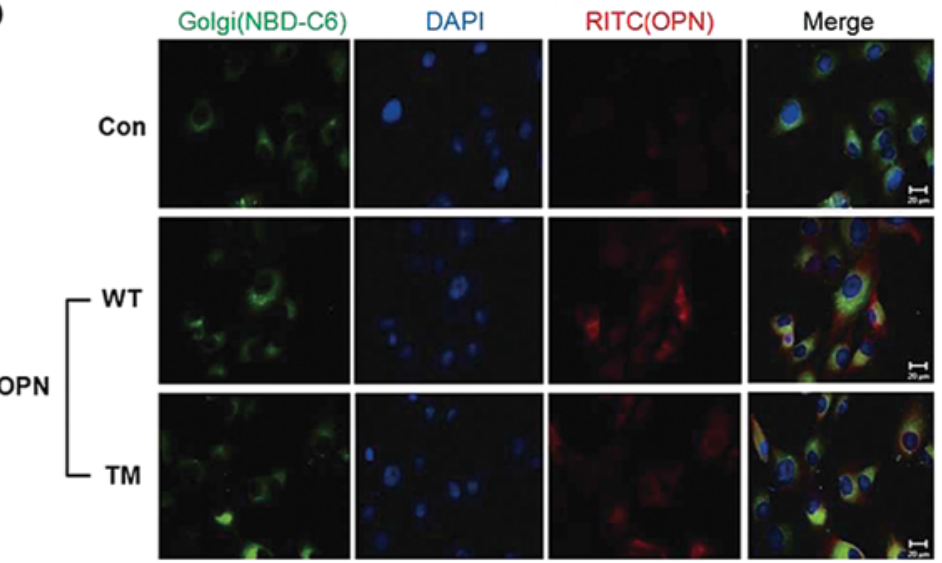

E

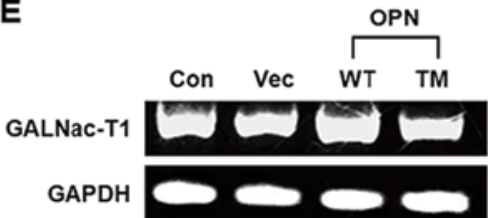

$\mathbf{F}$

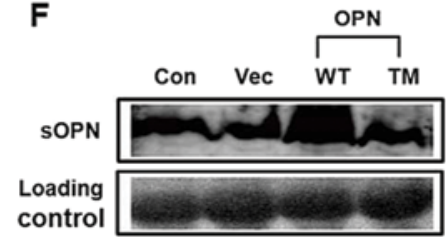

G

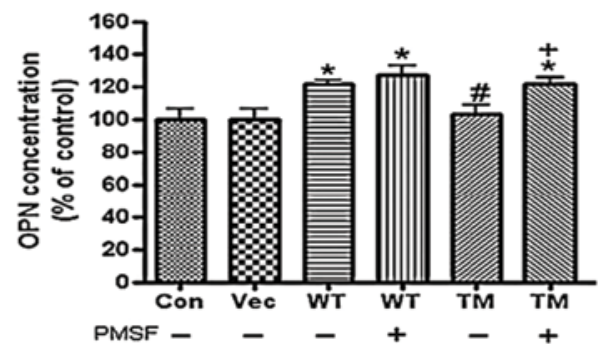

Figure 1. Expression of osteopontin (OPN) wild-type (WT) and triple mutant (TM) in H226 cells. (A) For the dual luciferase assay, the cells were co-transfected with different OPN constructs, as indicated, and with the bicistronic reporter construct pcDNAfLUC-polIRES-rLUC, and then lysed and assayed for firefly and renilla luciferase activities. Each bar presents the mean \pm SEM $(n=3)$. (B) Cells were co-transfected with OPN constructs, as indicated, and the NF- $\kappa B$ luciferase vector with the pRA-SV40 control vector, then lysed and assayed for firefly and renilla luciferase activities. (C) RT-PCR analysis of OPN. H226 cells were transfected with OPN WT/TM for $24 \mathrm{~h}$ and then the total RNA was isolated and subjected to RT-PCR. (D) Fluorescence imaging of OPN after the transfection. H226 cells were incubated with OPN WT and TM for $24 \mathrm{~h}$, followed by fixing and immunostaining for the OPN (red via TRITC), Golgi marker (green via NBD-C6 Ceramide) and nuclei (blue via DAPI). The scale bar is $20 \mu \mathrm{m}$. (E) RT-PCR analysis of GALNac-T1. H226 cells were transfected with OPN WT/ TM for $24 \mathrm{~h}$ and then the total RNA was isolated and subjected to RT-PCR. Representative bands are shown. (F) Western blotting of the secreted OPN. H226 cells were transected with OPN WT/TM and the cultured media were collected and subjected to western blot analysis. (G) ELISA of OPN. H226 cells were transfected with OPN WT/TM and then treated with PMSF $(0.5 \mathrm{mM})$, after which cultured media were collected and ELISA was performed. Each bar presents the mean \pm SEM (n=3). ${ }^{*} \mathrm{P}<0.05$ vs. Con, ${ }^{\prime} \mathrm{P}<0.05$ vs. WT $[\mathrm{PMSF}(-)]$, and ${ }^{+} \mathrm{P}<0.05$ vs. TM [PMSF (-)]. Con, control (H226 cells); Vec, vector control; WT, OPN WT-transfected cells; TM, OPN TM-transfected cells.

for the cytosol and nucleus (Fig. 5A). Furthermore, the Akt transfection enhanced the NF- $\mathrm{NB}$ activity in the stable cells that expressed OPN WT and TM (Fig. 5B). The expression of mTOR increased the expression levels of p-eIF4G and p-eIF4E (Fig. 5C) and the cap-dependent protein translation in the cells that stably expressed OPN WT and TM (Fig. 5D). The results also showed that silencing p70S6K decreased the expression levels of p-p70S6K and the cap-dependent protein translation in the cells that stably expressed OPN WT and TM (Fig. 5E and F).

Involvement of the mTOR and IKK signaling pathways in the OPN-mediated cap-dependent protein translation and $N F-\kappa B$ activity. The effects of different inhibitors on OPN-mediated protein translation and $N F-\kappa B$ activity were investigated. cis-Golgi was blocked to the medial-Golgi transport with monensin (20) and the expression levels of
p-Akt (Thr308) and p-eIF4E were examined by western blot analysis. It was found that the monensin treatment decreased the protein levels of p-Akt (Thr308) and p-eIF4E in the OPN WT/TM-transfected cells (Fig. 6A). Moreover, a significant decrease in the cap-dependent protein translation was observed in the OPN TM-transfected cells compared with the control. Monensin also reduced the OPN WT-mediated cap-dependent protein translation, as determined by the luciferase assay (Fig. 6B). To examine the role of mTOR and the involvement of the Akt/mTOR signaling pathway in OPN-regulated protein translation, the OPN WT/TM-transfected cells were treated with rapamycin and the expression levels of p-eIF4G and p-4E-BP1 were examined. The results showed that rapamycin decreased the expression levels of p-eIF4G and p-4E-BP1 in the OPN WT/TM-transfected cells (Fig. 6C). The luciferase assay clearly demonstrated that rapamycin attenuated the OPN-induced increase in the cap-dependent protein transla- 
A
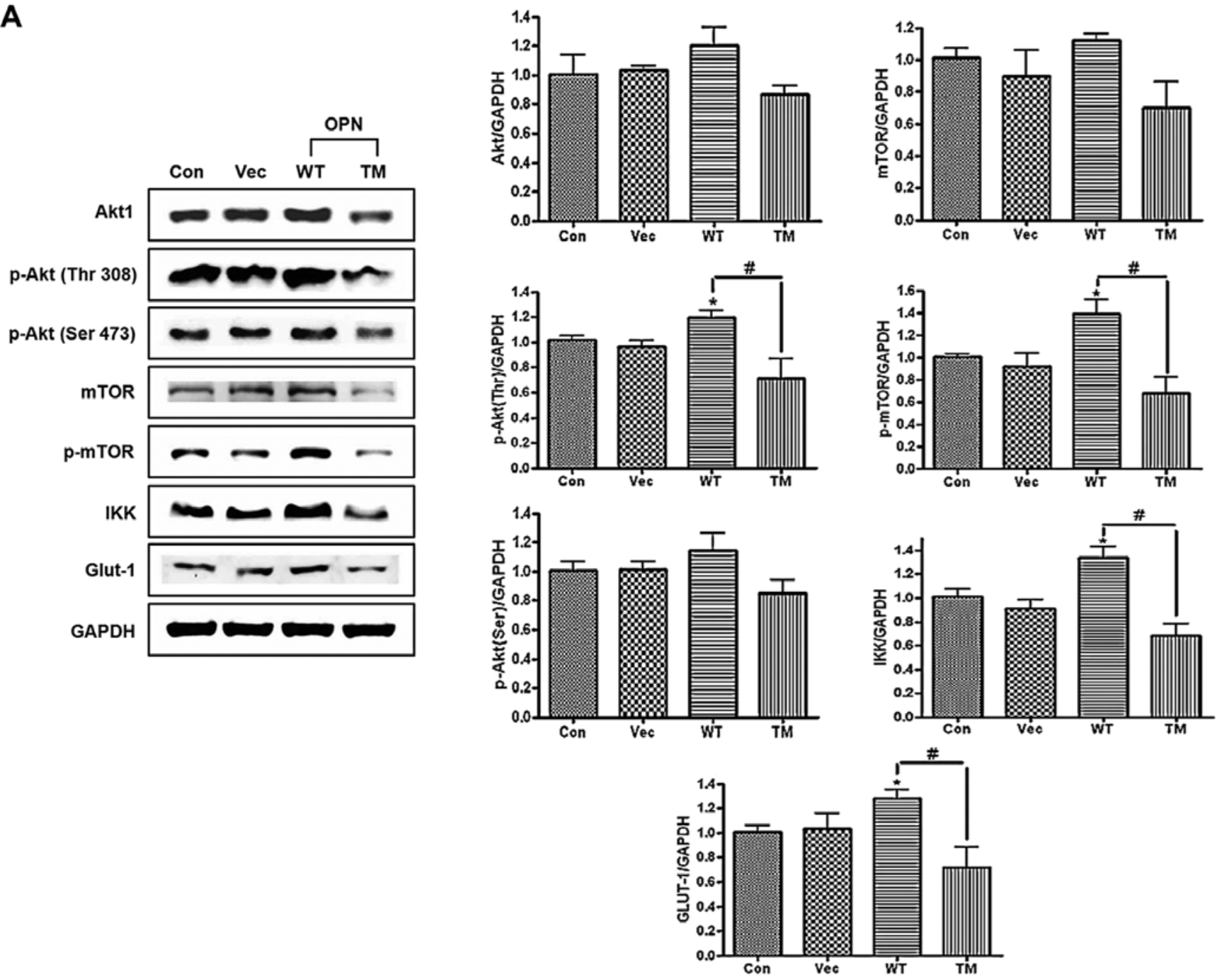

B

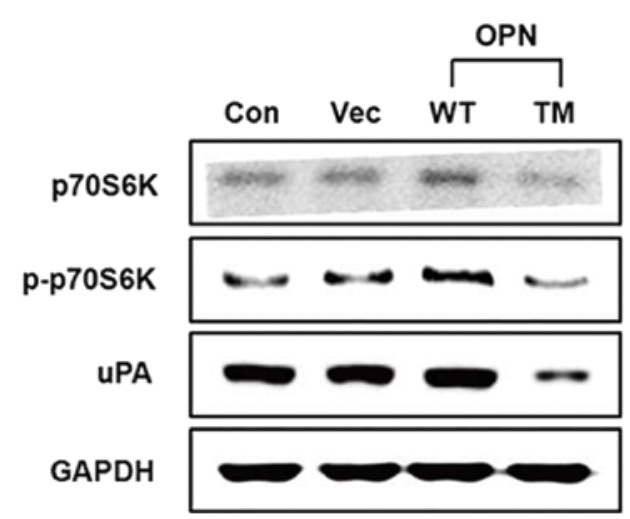

Figure 2. Western blot analysis of osteopontin (OPN)-dependent proteins. (A) Western blot analysis of Akt, p-Akt (Thr308), p-Akt (Ser473), mammalian target of rapamycin (mTOR), p-mTOR, IKK and Glut-1. H226 cells were transfected with OPN WT/TM and incubated for 48 h, followed by lysis and collection. The lysates were used for the western blotting. The right panel shows the results of the densitometric analysis of the bands of interest. Each bar presents the mean \pm SEM $(n=3)$. ${ }^{*} \mathrm{P}<0.05$ vs. Con and ${ }^{~} \mathrm{P}<0.05$ vs. TM. (B) Western blot analysis of p70S6K, p-p70S6K and uPA. H226 cells were transfected with osteopontin (OPN) wild-type (WT)/triple mutant (TM), as indicated earlier. The lysates were used for the western blotting.

tion. Moreover, a significant decrease was observed in the OPN TM-transfected cells following rapamycin treatment (Fig. 6D). To investigate whether OPN-mediated NF-kB activity occurs through the Akt/IKK signaling pathway, the PI3K inhibitor LY294002 and the IKK inhibitor PDTC were used.
The results showed that LY294002 and PDTC decreased the p-Akt (Thr308) and uPA expression levels, respectively, in the OPN WT/TM-transfected cells (Fig. 6E and G). The LY294002 and PDTC treatment decreased the protein level of NF- $\kappa$ B p65 in the cytosol and an increase in the NF- $\mathrm{kB}$ p65 expression was 

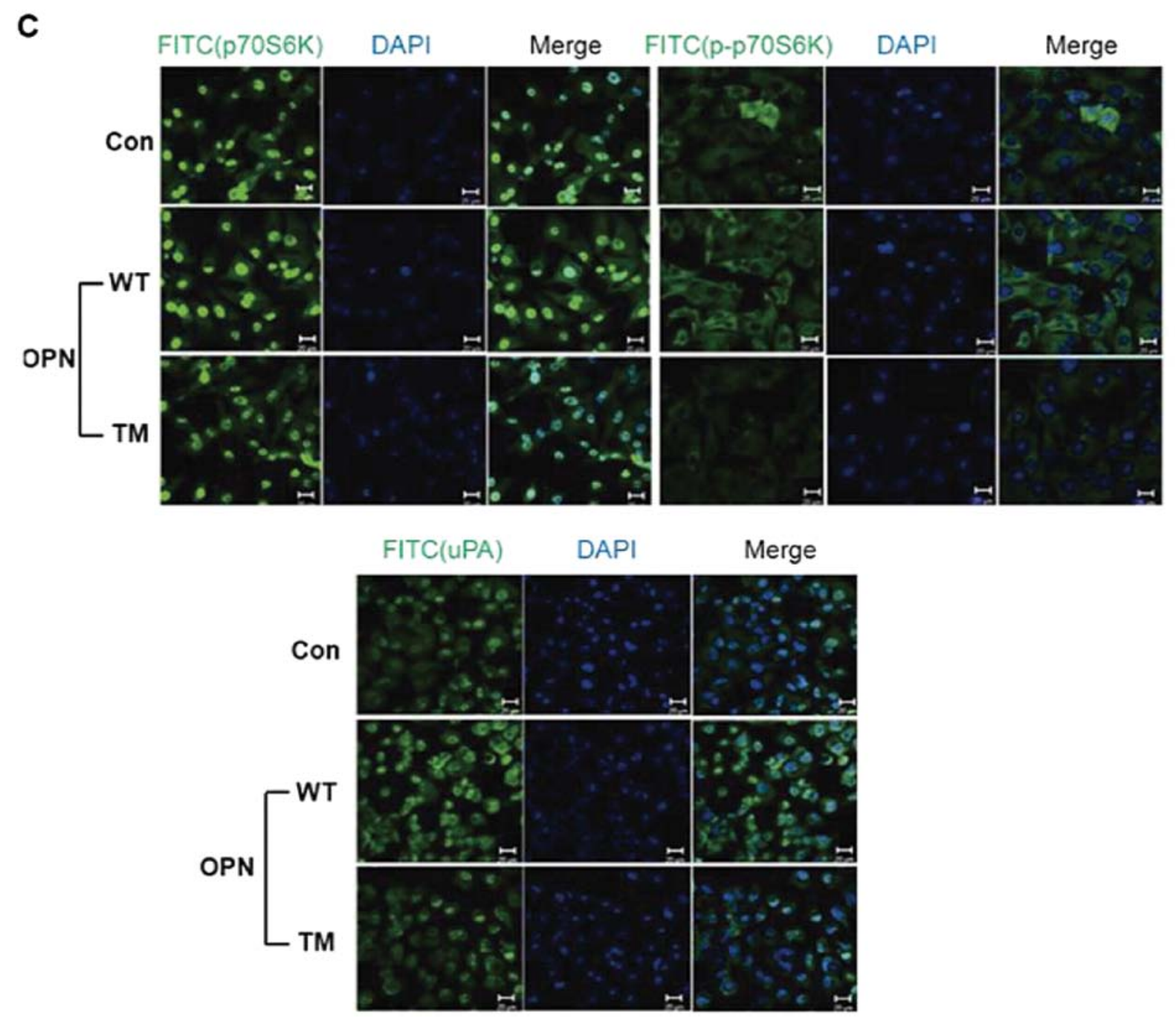

Figure 2. Continued. (C) (Upper panel) Fluorescence imaging of p-70S6K and p-p70S6K. (Lower panel) Fluorescence imaging of uPA. H226 cells were incubated with OPN WT and TM, followed by fixing and immunostaining for the indicated proteins (red via FITC) and nuclei (blue via DAPI). The scale bar is $20 \mu \mathrm{m}$. Con, control (H226 cells); Vec, vector control; WT, OPN WT-transfected cells; TM, OPN TM-transfected cells.

observed in the nucleus of the cells that were transfected with OPN WT and TM. Furthermore, PDTC or LY294002 inhibited the OPN-induced increase in NF- $\kappa \mathrm{B}$ activity and a significant decrease was observed in the OPN TM-transfected cells following treatment with LY294002 or PDTC (Fig. 6F and H).

Effect of OPN TM on the OPN-mediated signaling pathway in H322 cells. The effect of OPN TM on the NF- $\mathrm{BB}$ activity, cap-dependent protein translation, and OPN-mediated signaling pathway in $\mathrm{H} 322$ cells was also determined. The results showed that OPN TM decreased the protein levels of p-Akt, p-mTOR, p-eIF4G and p-eIF4E in the H322 cells (Fig. 7A). We also showed that OPN TM clearly decreased the NF- $\mathrm{NB}$ activity and cap-dependent protein translation, but that the PDTC and rapamycin treatment further decreased the cap-dependent protein translation and $\mathrm{NF}-\kappa \mathrm{B}$ activity in the OPN TM-transfected cells (Fig. 7B). These results suggest that OPN TM is capable of altering the OPN-dependent signaling pathway in H322 cells that reportedly have high levels of endogenous OPN (21).
Effects of OPN WT and TM on tumor growth in a xenograft mouse model. Evidence has shown that OPN increases tumor growth in several in vivo cancer models $(22,23)$. Due to the in vitro data obtained, we investigated the effects of OPN WT and TM on tumor growth in a xenograft mouse model. Accordingly nude mice were injected subcutaneously with $\mathrm{H} 226$ cells that were alone or stably transfected with OPN WT or TM. The data showed that OPN WT increased whereas OPN TM decreased the tumor growth and weight in the xenograft mouse model (Fig. 8A-C). It has been demonstrated that several OPN-dependent proteins, such as MMP-9, VEGF, FGF-2, COX-2 and uPA, are important in the angiogenesis, invasion and growth of tumor cells. Therefore, the expression levels of these proteins in a tumor xenograft model were investigated using western blotting and immunohistochemistry. The results showed that the expression of MMP-9, VEGF and FGF-2 increased in tumors generated with OPN WT-transfected cells but not with OPN TM-transfected cells, as determined by western blot and densitometric analyses (Fig. 8D). Furthermore, the IHC analysis showed a decrease 
A

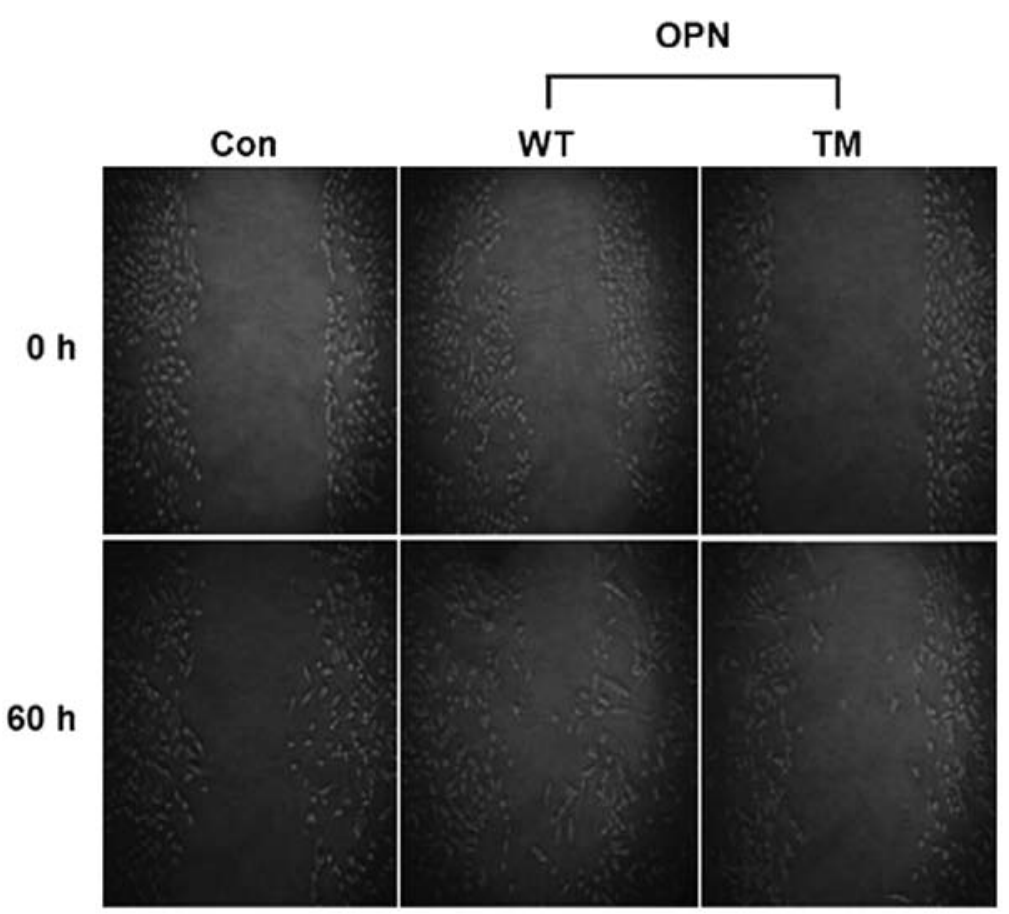

B

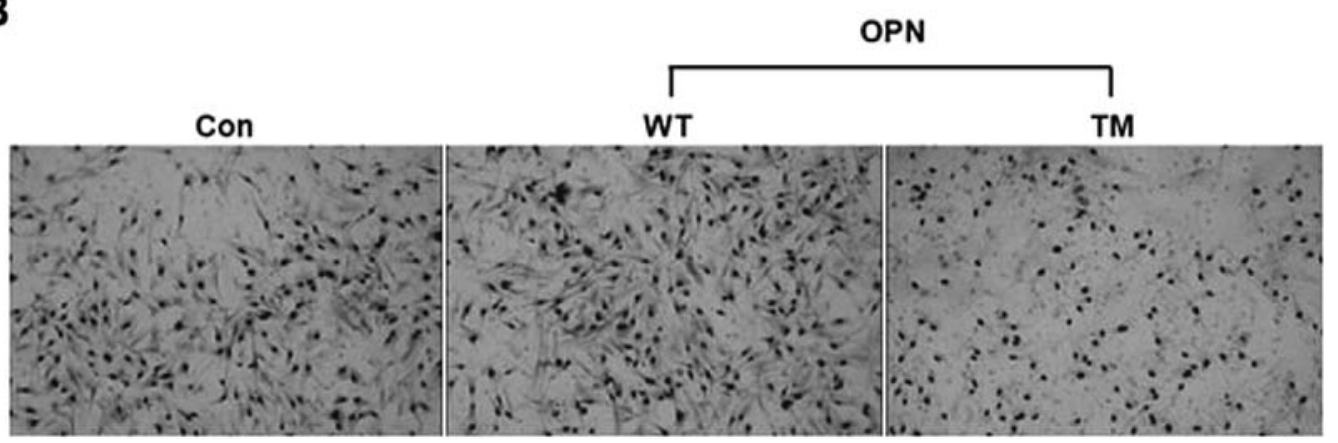

Figure 3. Wound healing and invasion assays. (A) Cell migration rate via wound healing in the H226 cells after the transfection. (B) Matrigel invasion assay of the H226 cells; representative images of the invading cells treated with osteopontin (OPN) wild-type (WT)/triple mutant (TM). Con, control (H226 cells); WT, OPN WT-transfected cells; TM, OPN TM-transfected cells.

in the expression levels of uPA and COX-2 in tumors generated with OPN TM-transfected cells and an increase in tumors generated with OPN WT-transfected cells (Fig. 8E).

\section{Discussion}

The abundance of clinical and experimental evidence regarding the relationship between OPN and lung cancer growth makes OPN an attractive potential therapeutic target for combating lung cancer development $(24,25)$. A specific OPN inhibitor is not yet available, however. Therefore, inhibition of the OPN-mediated signaling pathway is a potentially attractive strategy for lung cancer therapy.

The importance of the O-glycosylation sites in OPN and the connection between the OPN function and its glycosylation have yet to be adequately clarified. In the present study, the effect of the O-glycosylation sites in OPN on cancer cell growth and migration was functionally defined by introducing selective mutation in three O-glycosylation sites of OPN and expressing this mutation in lung cancer cells.
Overexpression of the OPN that contained WT glycosylation sites but not of that with the three mutated O-glycosylation sites increased lung cancer cell growth and migration in vitro as well as in a xenograft mouse model. Specifically, the specified O-glysylation sites were important for lung cancer progression and growth. Glycosylation is one of the most important post-translational events wherein glycan chains are connected to a specific peptide backbone. The uncertainty in predicting the correlation of OPN O-glycosylation with its function necessitates the examination of the relevance of O-glycosylation of OPN in its functional environment. Therefore, the extent of glycosylation in OPN must be determined, and the consequence of the blocking or modification of the glycosylation of OPN must be investigated (26-28). In the present study, this issue was investigated by studying the connection between $\mathrm{O}$-glycosylation and the cell function of OPN by expressing OPN TM and WT in lung cancer cells. The results clearly showed that OPN WT and TM increased the OPN mRNA levels. The overexpression of OPN in Golgi was also demonstrated via CLSM. 
A
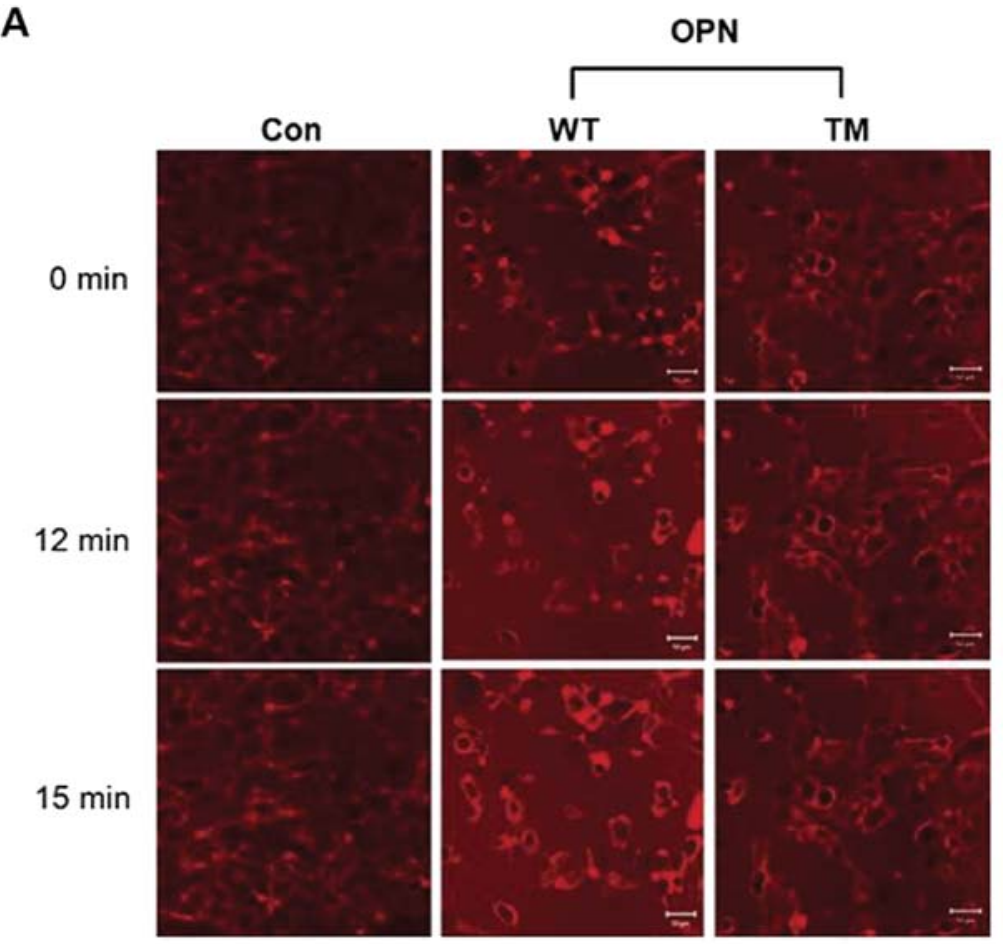

B

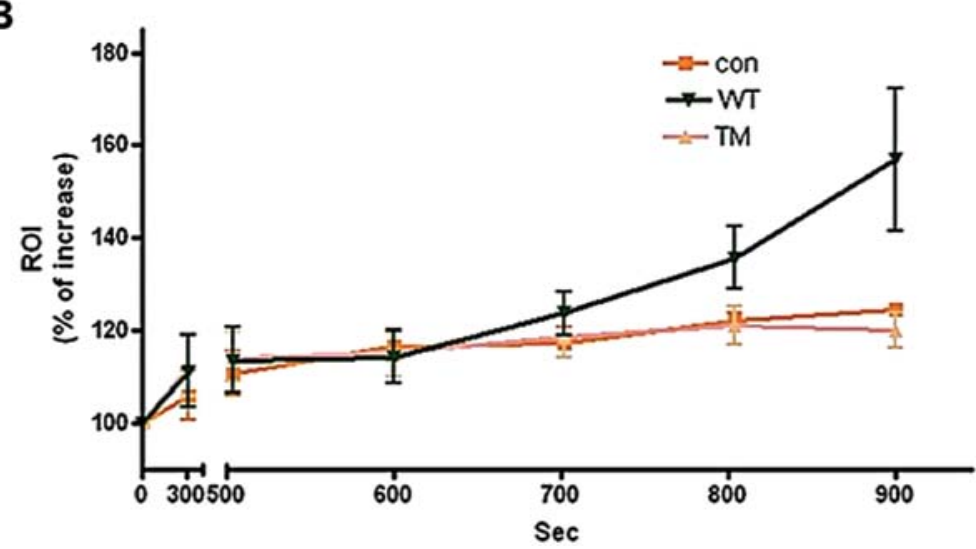

Figure 4. Cy3-Glc-a uptake by the H226 cells. (A) Cy3-Glc-a (6 $\mu \mathrm{M})$ added to the H226 cells that stably expressed osteopontin (OPN) wild-type (WT)/triple mutant (TM) and fluorescence images captured via live cell imaging with a confocal laser scanning microscopy (CLSM) are shown. (B) Fluorescence intensities determined via their continuous measurement from the regions of interest in three independent cells. Con, control (H226 cells); WT, cells that stably expressed OPN WT; TM, Cells that stably expressed OPN TM.

The transfer of $\alpha-\mathrm{N}$-acetylgalactosamine (GalNAc) from UDP-GalNAc to Ser or Thr is catalyzed by UDP-GalNAc:polypeptide $\mathrm{N}$-acetylgalactosaminyltransferases (GalNAc-Ts) in Golgi (29,30). Miwa et al (11) determined the O-glycosylation sites of OPN as potential acceptor substrates of GalNAc-T1 in vitro and demonstrated that two of its glycosylation sites, which are also conserved in humans, were glycosylated by GalNAc-T1. The results showed that OPN WT increased the expression levels of GalNAc-T1, whereas OPN TM did not, suggesting that the introduction of OPN regulates the expression of O-glycosylation-related proteins in the Golgi, which can affect its $\mathrm{O}$-glycosylation process and suggests that unlike OPN WT, OPN TM cannot be fully glycosylated. It has been reported that PTMs that include O-glycosylation can affect protein conformation, stability and other properties (31-34). Glycosylation modification has been reported to protect proteins against proteasomal degradation (35-37).
Unlike OPN WT, OPN TM did not increase the expression of the secreted OPN; however, when the cells were treated with PMSF, the expression levels of OPN were recovered. The data clearly show that non-fully-glycosylated OPN, in contrast to fully glycosylated OPN, generates different functional forms that may alter their physiological functions. Mutations at specific O-glycosylation sites of OPN may generally result in lower protein stability, indicating that there is a correlation between alteration in OPN O-glycosylation and its stability against proteasome degradation. The results clearly show that unlike OPN WT, OPN TM did not increase Akt and p-Akt at the phosphorylation sites. It has been reported that OPN is able to induce Akt and that its related signals contribute to cancer progression $(1,38)$.

Translational control, a crucial element in the regulation of gene expression, has been reported to be frequently deregulated in cancer cells (39-41). Translation is regulated 
A

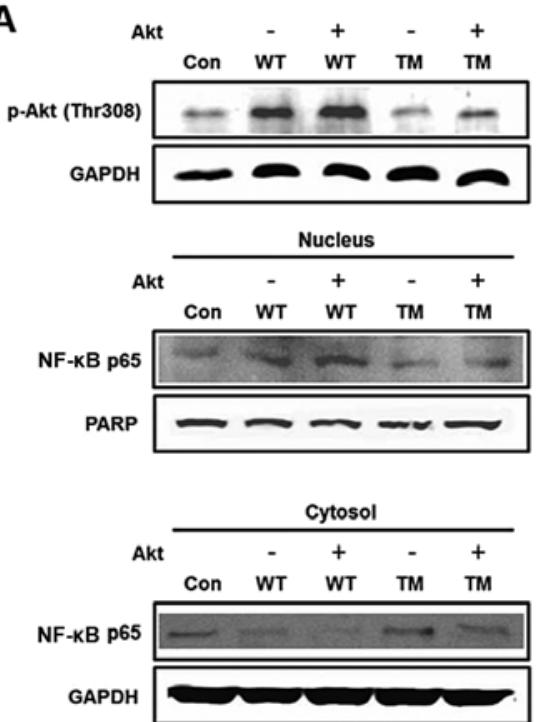

C

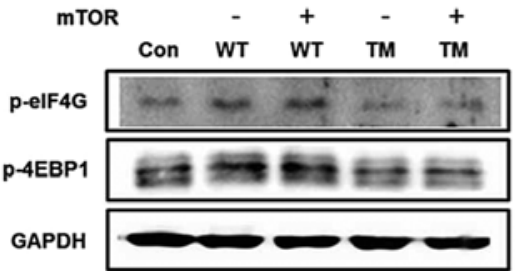

D

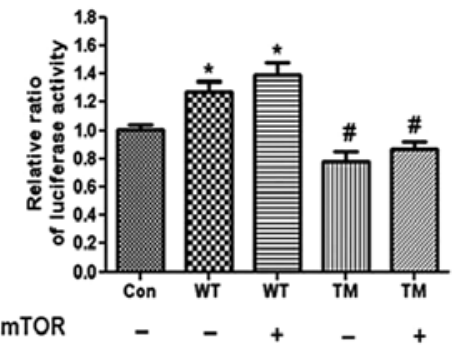

E

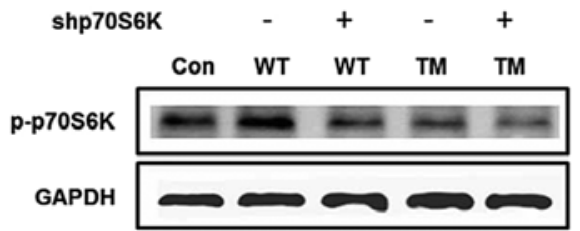

$\mathbf{F}$

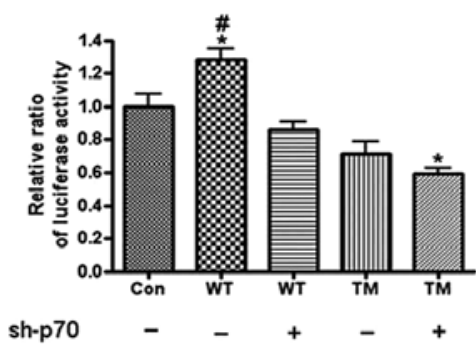

Figure 5. Western blot analysis of the proteins that mediated the Akt/IKK and protein synthesis signaling pathway. (A) Cells that stably expressed the osteopontin (OPN) wild-type (WT)/triple mutant (TM) transfected with the Akt expression vector and then subjected to western blot analysis for p-Akt (Thr308). (Lower panel) Western blot analysis of NF- $\mathrm{kB}$ p65 in the cytosol and nucleus. The cells were collected and then separated into cytosolic and nucleic fractions using a Nuclear Extract kit. GAPDH and PARP were used as controls for the cytosol and nucleus, respectively. (B) For the dual luciferase assay, cells that stably expressed OPN WT/TM were co-transfected with Akt and the NF- $\kappa \mathrm{B}$ luciferase vector with the pRA-SV40 control vector and then assayed for firefly and renilla luciferase activities. Each bar presents the mean $\pm \mathrm{SEM}(\mathrm{n}=3)$. ${ }^{*} \mathrm{P}<0.05$ vs. Con and ${ }^{\#} \mathrm{P}<0.05$ vs. WT+Akt and WT. Con, control (H226 cells); WT, cells that stably expressed OPN WT; TM, cells that stably expressed OPN TM. (C) Cells that stably expressed the OPN WT/TM that was transfected with the mammalian target of rapamycin (mTOR) expression vector and then subjected to western blot analysis for p-eIF4G and p-4EBP-1. (D) For the dual luciferase assay, the cells that stably expressed the OPN WT/TM were co-transfected with mTOR and bicistronic reporter constructs, and then assayed for firefly and renilla luciferase activities. Each bar presents the mean \pm SEM $(n=3)$. ${ }^{*} \mathrm{P}<0.05$ vs. Con and ${ }^{\#} \mathrm{P}<0.05$ vs. WT+mTOR and WT. Con, control (H226 cells); WT, cells that stably expressed the OPN WT; TM, cells that stably expressed the OPN TM. (E) Cells that stably expressed the OPN WT/TM that was infected with lentivirus which contained the shRNA that targeted p70S6K $(10 \mathrm{ng} / \mathrm{ml})$ and were then subjected to western blot analysis for p-p70S6K. (F) For the dual luciferase assay, cells that stably expressed the OPN WT/TM were infected and transfected with bicistronic reporter constructs and then assayed for firefly and renilla luciferase activities. Each bar presents the mean \pm SEM $(n=3)$. ${ }^{*} \mathrm{P}<0.05$ vs. Con and ${ }^{\#} \mathrm{P}<0.05$ vs. other treated groups. Con, control (H226 cells); WT, cells that stably expressed the OPN WT; TM, cells that stably expressed the OPN TM.

via the mTOR signaling pathway through p70S6K and 4E-BP1/eIF4E, wherein OPN-activated Akt can be a crucial intermediate. A key step in the regulation of protein synthesis is the assembly of the initiation complex eIF4F that contains the initiation factors eIF4G, eIF4E (the protein that binds to the $5^{\prime}$ cap structure) and eIF4A. 4E-BP1 has been reported to bind to a site on eIF4E that overlaps with the binding site for eIF4G (42-44). Thus, 4E-BP1 competes with eIF4G in order to bind to eIF4E, and is thus able to inhibit the eIF4F complex formation. Phosphorylation of 4E-BP1 reduces its affinity to eIF4E and its dissociation, making eIF4E available for binding to eIF4G and thereby increasing the mRNA translation $(45,46)$. The results have shown that OPN TM decreased the expression levels of mTOR, p-mTOR, p70S6K, p-p70S6K, p-4E-BP1, p-eIF4E and p-eIF4G, but increased the expression levels in the OPN WT-treated cells. The results also showed that the expression of mTOR induced the cap-dependent protein translation, whereas rapamycin and shp70S6K inhibited the cap-dependent protein translation in the cells treated with OPN WT and TM. These data reveal that OPN WT induced cap-dependent protein translation through the Akt-mTOR pathway, and that OPN TM inhibits cap-dependent protein translation which can affect lung cancer cell growth.

Recent studies have indicated that OPN plays a crucial role in controlling cell motility. Moreover, NF- $\kappa \mathrm{B}$ activation has been reported to be a major effector of OPN-mediated cell migration and motility $(47,48)$. In an inactivated state, $\mathrm{NF}-\kappa \mathrm{B}$ is usually kept in the cytoplasm by the inhibitor protein $\mathrm{I} \kappa \mathrm{B}$. Upon the $\mathrm{NF}-\kappa \mathrm{B}$ stimulation, the $\mathrm{I} \kappa \mathrm{B}$ proteins are phosphorylated by the multi-sub-unit IKK complex, which subsequently targets $\mathrm{I} \kappa \mathrm{B}$ for ubiquitination. Additionally, the free $N F-\kappa B$ translocates to the nucleus and activates the target genes (49). The results show that OPN WT increased NF- $\mathrm{BB}$ 


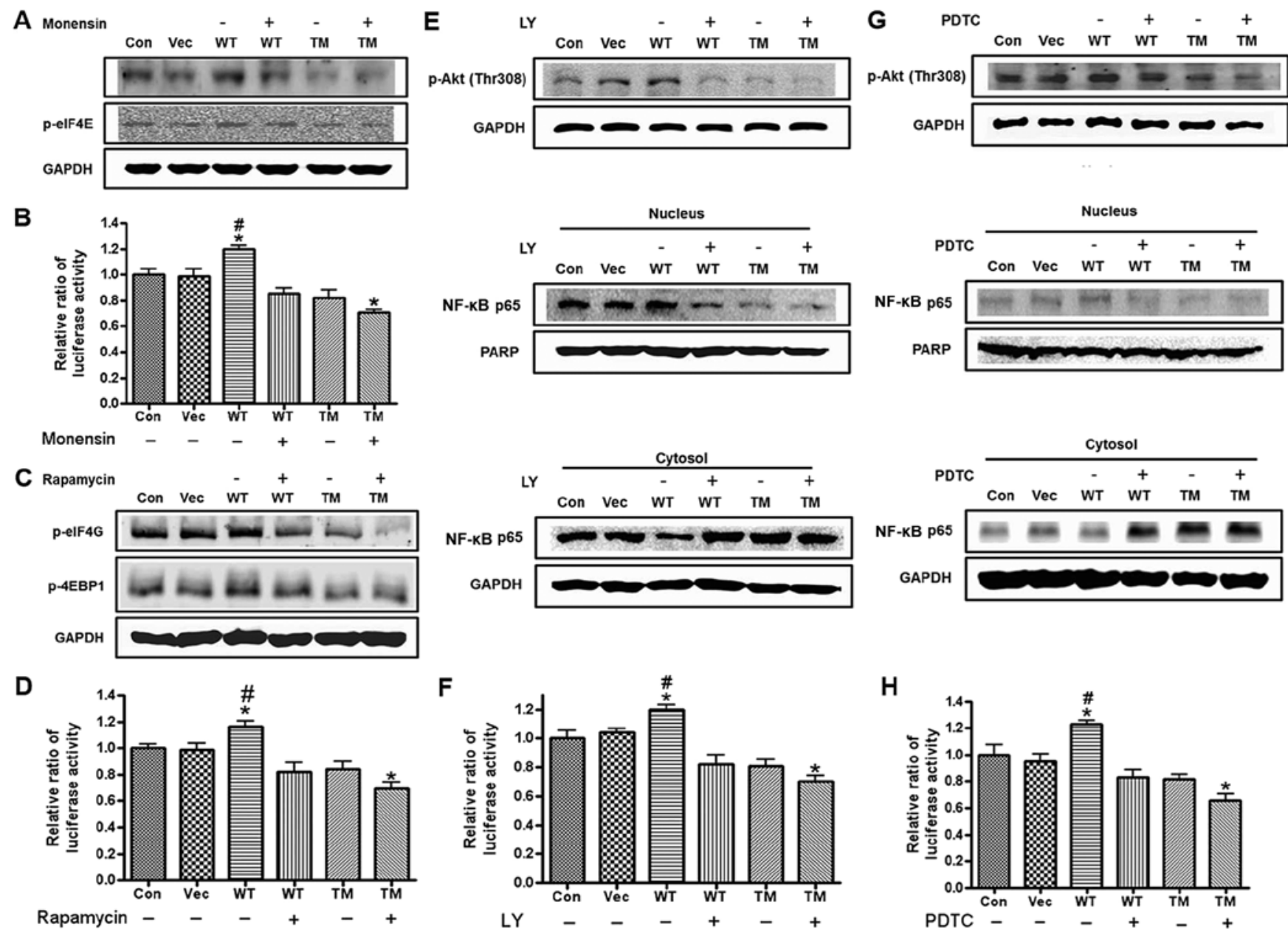

Figure 6. Effect of monensin, rapamycin, LY294402 and PDTC in cells transfected with osteopontin (OPN) wild-type (WT) and triple mutant (TM). (A) Western blot analysis of p-Akt (Thr308) and p-eIF4E. H226 cells were transfected with OPN WT/TM and then treated with monensin $(0.5 \mathrm{mM})$. The lysates were used for the western blotting. (B) For the dual luciferase assay, cells were co-transfected with OPN constructs (vector only, OPN WT and OPN TM) and the bicistronic reporter construct pcDNAfLUC-polIRES-rLUC and then treated with monensin or left untreated. The cells were lysed and assayed for firefly and renilla luciferase activities. Each bar presents the mean \pm SEM $(n=3)$. ${ }^{*} \mathrm{P}<0.05$ vs. Con and ${ }^{\#} \mathrm{P}<0.05$ vs. other treated groups. Con, control (H226 cells); Vec, vector control; WT, OPN WT-transfected cells; TM, OPN TM-transfected cells. (C) Western blot analysis of p-4E-BP1 and p-eIF4G. H226 cells were transfected with OPN WT/TM and then treated with rapamycin $(100 \mathrm{nM})$. The lysates were used for the western blotting. (D) For the dual luciferase assay, the cells were co-transfected with OPN constructs (vector only, OPN WT and OPN TM) and the bicistronic reporter construct pcDNAfLUC-polIRES-rLUC, and then treated with rapamycin or left untreated. The cells were then lysed and assayed for firefly and renilla luciferase activities. Each bar presents the mean \pm SEM ( $\mathrm{n}=3$ ). ${ }^{*} \mathrm{P}<0.05$ vs. Con and ${ }^{\#} \mathrm{P}<0.05$ vs. other treated groups. Con, control (H226 cells); Vec, vector control; WT, OPN WT-transfected cells; TM, OPN TM-transfected cells. (E) Western blot analysis of p-Akt (Thr308). H226 cells were transfected with OPN WT/TM and then treated with LY294402 (100 nM). The lysates were used for the western blotting. (Lower panel) Western blot analysis of NF- $\mathrm{kB}$ p65 in the cytosol and nucleus. The cells were collected and separated into cytosolic and nucleic fractions using a Nuclear Extract kit. GAPDH and PARP were used as controls for the cytosol and nucleus, respectively. (F) For the dual luciferase assay, the cells were co-transfected with OPN constructs (vector only, OPN WT and OPN TM) and the NF-kB luciferase vector with the pRA-SV40 control vector and then treated with LY294002 or left untreated. The cells were then lysed and assayed for firefly and renilla luciferase activities. Each bar presents the mean \pm SEM $(n=3)$. ${ }^{*} \mathrm{P}<0.05$ vs. Con and ${ }^{\#} \mathrm{P}<0.05$ vs. other treated groups. Con, control $(\mathrm{H} 226$ cells); Vec, vector control; WT, OPN WT-transfected cells; TM, OPN TM-transfected cells. (G) Western blot analysis of uPA. H226 cells were transfected with OPN WT/TM and then treated with LY294402 (100 nM). The lysates were used for the western blotting. (Lower panel) Western blot analysis of NF- $\mathrm{kB}$ p65 in the cytosol and nucleus. The cells were collected and then separated into cytosolic and nucleic fractions using a Nuclear Extract kit. GAPDH and PARP were used as controls for the cytosol and nucleus, respectively. (H) For the dual luciferase assay, the cells were co-transfected with OPN constructs (vector only, OPN WT and OPN TM) and the NF- $\mathrm{KB}$ luciferase vector with the pRA-SV40 control vector and then treated with PDTC or left untreated. The cells were then lysed and assayed for firefly and renilla luciferase activities. Each bar presents the mean \pm SEM $(n=3)$. " $\mathrm{P}<0.05$ vs. Con and ${ }^{~} \mathrm{P}<0.05$ vs. other treated groups. Con, control (H226 cells); Vec, vector control; WT, OPN WT-transfected cells; TM, OPN TM-transfected cells.

activity, which enhanced the motility of the lung cancer cells by activating Akt, IKK, NF- $\mathrm{B}$ p-65 and uPA, unlike OPN TM. By contrast, treatment of the cells with LY294002 and PDTC reduced the NF- $\kappa \mathrm{B}$ activity induced by OPN WT and caused a further decrease in the cells transfected with OPN $\mathrm{TM}$, whereas the expression of Akt increased NF- $\mathrm{BB}$ activity, suggesting that an OPN WT-induced increase in cell motility through the $\mathrm{NF}-\kappa \mathrm{B}$ signaling pathway can be antagonized by the introduction of OPN TM.
Glucose is the most important energy source for cell growth. Rapidly growing cancer cells need more glucose than normal cells. Glucose passage across the cell membrane is regulated by a family of glucose transporters (Gluts). In non-small-cell lung cancer (NSCLC), glucose uptake is mediated by Glut-1, a significant indicator of poor prognosis in NSCLC $(50,51)$. It has been reported that the $N F-\kappa B$ pathway controls Glut-1 trafficking and activation (52). In the present study, a novel fluorescence-labeled glucose analogue, Cy3-linked 
A

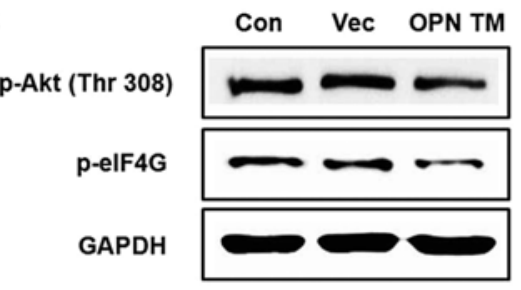

B
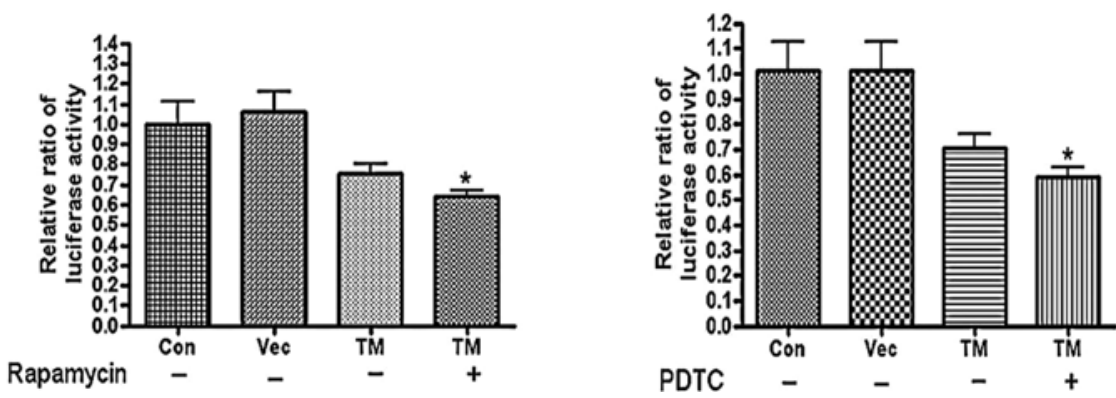

Figure 7. Effects of osteopontin (OPN) triple mutant (TM) on H322 cells. (A) Western blot analysis of p-Akt (Thr308), p-mTOR, p-eIF4E and p-eIF4G. The H322 cells were transfected with OPN TM and the lysates were used for the western blotting. Representative bands are shown. (B) (Right panel) For the dual luciferase assay, the cells were co-transfected with OPN TM and the bicistronic reporter construct pcDNAfLUC-polIRES-rLUC and then treated with rapamycin or left untreated. The cells were then lysed and assayed for firefly and renilla luciferase activities. (Left panel) For the dual luciferase assay, the cells were co-transfected with OPN TM and the NF-кB luciferase vector with the pRA-SV40 control vector and then treated with PDTC or left untreated. The cells were then lysed and assayed for firefly and renilla luciferase activities. Each bar presents the mean \pm SEM ( $n=3)$. " $\mathrm{P}<0.05$ vs. Con. Con, control (H322 cells); Vec, vector control; TM, OPN TM-transfected cells.

A
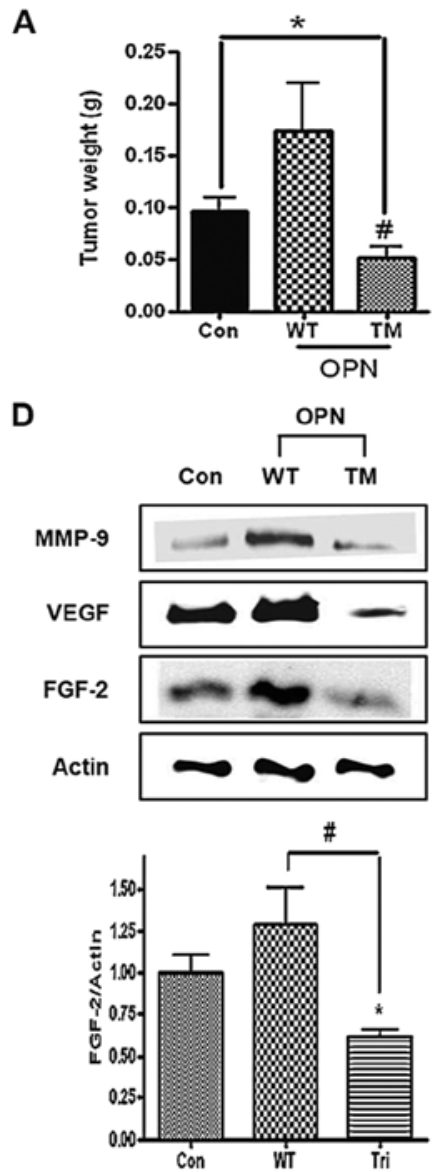

B

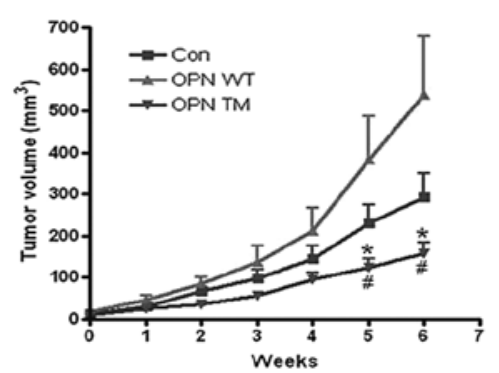

E
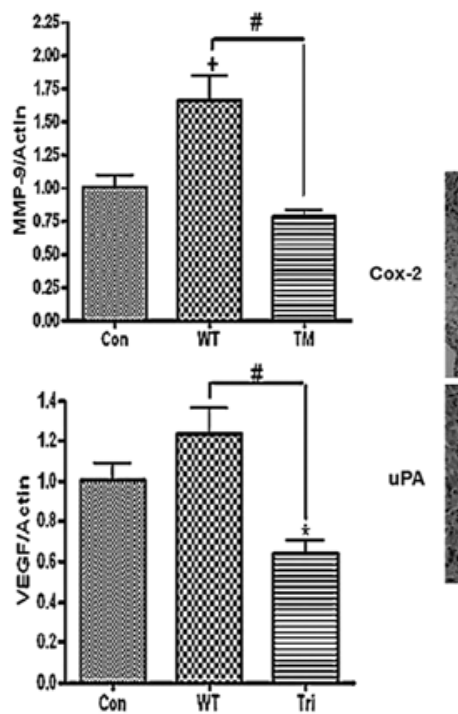

UPA
C

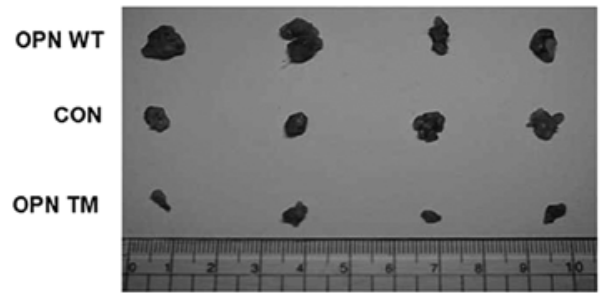

Con

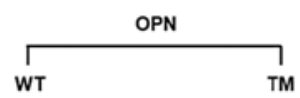

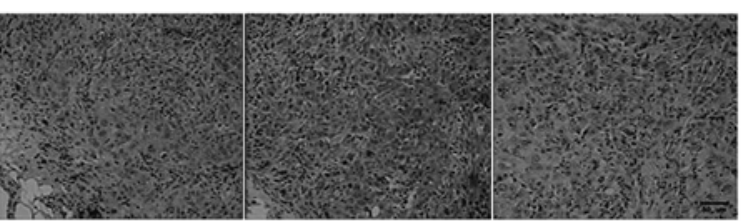

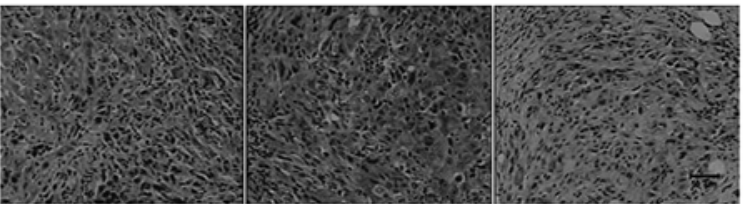

Figure 8. Effects of osteopontin (OPN) wild-type (WT) and triple mutant (TM) on tumor growth, angiogenesis, migration and invasion in xenograft model mice. Nude mice inoculated subcutaneously with H226 cells that were either non-transfected or stably transfected with OPN WT or OPN TM. (A) Bar graph of the measured tumor weights. Each bar presents the mean \pm SEM $(n=7)$. ${ }^{*}<<0.05$ vs. Con and ${ }^{\#} \mathrm{P}<0.05$ vs. WT. (B) The tumor growth was monitored and the volumes were calculated, as described in Materials and methods. ${ }^{*} \mathrm{P}<0.05$ compared to Con and ${ }^{\#} \mathrm{P}<0.05$ compared to WT. (C) Representative image of the tumors in the nude mice. Con, control; WT, OPN WT xenograft tumor; TM, OPN TM xenograft tumor. Tumor homogenates subjected to western blot analysis. The blots were probed with antibodies, as indicated. (D) Expression levels of MMP-9, VEGF and FGF-2. The right panel shows the results of the densitometric analysis of the bands of interest. Each bar presents the mean \pm SEM $(n=3)$. ${ }^{*}<0.05$ vs. Con and ${ }^{\#} \mathrm{P}<0.05$ vs. TM. (E) Immunohistochemical analysis of COX-2 and $u$ PA (magnification, $\mathrm{x} 200$; bar $=50 \mu \mathrm{m}$; Con, control; WT, OPN WT xenograft tumor; TM, OPN TM xenograft tumor). 
O-1-glycosylated glucose, was used, which can behave as D-glucose to measure the glucose uptake. Cy3-labeled glucose is tolerant to intense light sources and can be applied in a bioassay system without glucose starvation (53). The results of the present study show that OPN TM did not increase the Glut-1 expression and the glucose uptake, which may decrease the glucose consumption rate, unlike OPN WT.

The decreased expression of several proteins associated with angiogenesis, invasion, and migration, such as MMP-9, FGF-2, VEGF, uPA and COX-2, in the OPN TM xenograft tumor suggests that OPN TM may reduce tumor progression and growth, whereas the increased expression levels of the indicated proteins increased tumor growth in the OPN WT xenograft mouse model.

Taken together, the results have shown that unlike OPN WT, OPN TM did not induce Akt-mediated cap-dependent protein translation and NF- $\kappa B$ activity. Overexpression of the TM of OPN may compete with endogenous OPN for substrates that can be limiting, which may prevent endogenous OPN from performing its proper function. Therefore, targeting OPN and regulating the OPN signaling and function by influencing post-translational glycosylation through the introduction of an inactive mutant of OPN can control lung cancer growth and migration and may have a useful application in the treatment of lung cancer.

\section{Acknowledgements}

The present study was supported by the National Research Foundation grants (NRF-2009-0078641 and 2012M3A9C4048819) from the Ministry of Education, Science and Technology (MEST). M.H.C. was also partially supported by the Research Institute for Veterinary Science, Seoul National University.

\section{References}

1. Donati V, Boldrini L, Dell'Omodarme M, et al: Osteopontin expression and prognostic significance in non-small cell lung cancer. Clin Cancer Res 11: 6459-6465, 2005.

2. Ahmed M, Behera R, Chakraborty G, et al: Osteopontin: a potentially important therapeutic target in cancer. Expert Opin Ther Targets 15: 1113-1126, 2011.

3. Weber GF: The metastasis gene osteopontin: a candidate target for cancer therapy. Biochim Biophys Acta 1552: 61-85, 2001.

4. Shevde LA, Das S, Clark DW and Samant RS: Osteopontin: an effector and an effect of tumor metastasis. Curr Mol Med 10: 71-81, 2010.

5. Chellaiah MA, Biswas RS, Rittling SR, Denhardt DT and Hruska KA: Rho-dependent Rho kinase activation increases CD44 surface expression and bone resorption in osteoclasts. J Biol Chem 278: 29086-29097, 2003.

6. Christensen B, Kazanecki CC, Petersen TE, Rittling SR, Denhardt DT and Sørensen ES: Cell type-specific post-translational modifications of mouse osteopontin are associated with different adhesive properties. J Biol Chem 282: 19463-19472, 2007.

7. Fukuda M: Roles of cell surface carbohydrates in cell adhesion; in particular those for sialyl Lewis $\mathrm{x}$ and polysialic acid. Seikagaku 72: 269-283, 2000 (In Japanese).

8. López-Ferrer A, Barranco C and de Bolós C: Differences in the $\mathrm{O}$-glycosylation patterns between lung squamous cell carcinoma and adenocarcinoma. Am J Clin Pathol 118: 749-755, 2002.

9. Christensen B, Nielsen MS, Haselmann KF, Petersen TE and Sørensen ES: Post-translationally modified residues of native human osteopontin are located in clusters: identification of 36 phosphorylation and five O-glycosylation sites and their biological implications. Biochem J 390: 285-292, 2005.
10. Keykhosravani M, Doherty-Kirby A, Zhang C, Brewer D, Goldberg HA, Hunter GK and Lajoie G: Comprehensive identification of post-translational modifications of rat bone osteopontin by mass spectrometry. Biochemistry 44: 6990-7003, 2005.

11. Miwa HE, Gerken TA, Jamison O and Tabak LA: Isoform-specific $\mathrm{O}$-glycosylation of osteopontin and bone sialoprotein by polypeptide $\mathrm{N}$-acetylgalactosaminyltransferase-1. J Biol Chem 285: 1208-1219, 2010

12. Aggarwal BB, Vijayalekshmi RV and Sung B: Targeting inflammatory pathways for prevention and therapy of cancer: short-term friend, long-term foe. Clin Cancer Res 15: 425-430, 2009.

13. Aggarwal BB and Sung B: NF- $\kappa$ B in cancer: a matter of life and death. Cancer Discov 1: 469-471, 2011.

14. Chaturvedi MM, Sung B, Yadav VR, Kannappan R and Aggarwal BB: NF- $\kappa \mathrm{B}$ addiction and its role in cancer: 'one size does not fit all'. Oncogene 30: 1615-1630, 2011

15. Ruggero D and Pandolfi PP: Does the ribosome translate cancer? Nat Rev Cancer 3: 179-192, 2003.

16. Abraham RT and Eng CH: Mammalian target of rapamycin as a therapeutic target in oncology. Expert Opin Ther Targets 12: 209-222, 2008.

17. Hagner PR, Schneider A and Gartenhaus RB: Targeting the translational machinery as a novel treatment strategy for hematologic malignancies. Blood 115: 2127-2135, 2010.

18. Kim YK, Kwon JT, Choi JY, et al: Suppression of tumor growth in xenograft model mice by programmed cell death 4 gene delivery using folate-PEG-baculovirus. Cancer Gene Ther 17: 751-760, 2010.

19. Minai-Tehrani A, Jiang HL, Kim YK, et al: Suppression of tumor growth in xenograft model mice by small interfering RNA targeting osteopontin delivery using biocompatible poly(amino ester). Int J Pharm 431: 197-203, 2012.

20. Tartakoff AM: Perturbation of vesicular traffic with the carboxylic ionophore monensin. Cell 32: 1026-1028, 1983.

21. Shijubo N, Uede T, Kon S, et al: Vascular endothelial growth factor and osteopontin in stage I lung adenocarcinoma. Am J Respir Crit Care Med 160: 1269-1273, 1999.

22. Philip S, Bulbule A and Kundu GC: Osteopontin stimulates tumor growth and activation of promatrix metalloproteinase-2 through nuclear factor-kappa B-mediated induction of membrane type 1 matrix metalloproteinase in murine melanoma cells. J Biol Chem 276: 44926-44935, 2001.

23. Jain S, Chakraborty G and Kundu GC: The crucial role of cyclooxygenase-2 in osteopontin-induced protein kinase $\mathrm{C}$ alpha/c-Src/ IkappaB kinase alpha/beta-dependent prostate tumor progression and angiogenesis. Cancer Res 66: 6638-6648, 2006.

24. Rodrigues LR, Teixeira JA, Schmitt FL, Paulsson M and Lindmark-Mänsson $\mathrm{H}$ : The role of osteopontin in tumor progression and metastasis in breast cancer. Cancer Epidemiol Biomarkers Prev 16: 1087-1097, 2007.

25. Xue YH, Zhang XF, Dong QZ, et al: Thrombin is a therapeutic target for metastatic osteopontin-positive hepatocellular carcinoma. Hepatology 52: 2012-2022, 2010.

26. Chang VT, Crispin M, Aricescu AR, et al: Glycoprotein structural genomics: solving the glycosylation problem. Structure 15: 267-273, 2007.

27. Patsos G, Robbe-Masselot C, Klein A, et al: O-glycan regulation of apoptosis and proliferation in colorectal cancer cell lines. Biochem Soc Trans 35: 1372-1374, 2007.

28. Varki A: Glycan-based interactions involving vertebrate sialic-acid-recognizing proteins. Nature 446: 1023-1029, 2007.

29. Ten Hagen KG, Tetaert D, Hagen FK, et al: Characterization of a UDP-GalNAc: polypeptide N-acetylgalactosaminyltransferase that displays glycopeptide $\mathrm{N}$-acetylgalactosaminyltransferase activity. J Biol Chem 274: 27867-27874, 1999.

30. Perrine CL, Ganguli A, Wu P, et al: Glycopeptide-preferring polypeptide GalNAc transferase 10 (ppGalNAc T10), involved in mucin-type O-glycosylation, has a unique GalNAc-O-Ser/ Thr-binding site in its catalytic domain not found in ppGalNAc T1 or T2. J Biol Chem 284: 20387-20397, 2009.

31. Han I and Kudlow JE: Reduced O glycosylation of Sp1 is associated with increased proteasome susceptibility. Mol Cell Biol 17: 2550-2558, 1997.

32. Lin YH and Yang-Yen HF: The osteopontin-CD44 survival signal involves activation of the phosphatidylinositol 3-kinase/Akt signaling pathway. J Biol Chem 276: 46024-46030, 2001.

33. Mann M and Jensen ON: Proteomic analysis of post-translational modifications. Nat Biotechnol 21: 255-261, 2003. 
34. Kazanecki CC, Uzwiak DJ and Denhardt DT: Control of osteopontin signaling and function by post-translational phosphorylation and protein folding. J Cell Biochem 102: 912-924, 2007.

35. Jackson SP and Tjian R: O-glycosylation of eukaryotic transcription factors: implications for mechanisms of transcriptional regulation. Cell 55: 125-133, 1988.

36. Gasbarri A, Del Prete F, Girnita L, Martegani MP, Natali PG and Bartolazzi A: CD44s adhesive function spontaneous and PMA-inducible CD44 cleavage are regulated at post-translational level in cells of melanocytic lineage. Melanoma Res 13: 325-337, 2003.

37. Solá RJ and Griebenow K: Effects of glycosylation on the stability of protein pharmaceuticals. J Pharm Sci 98: 1223-1245, 2009.

38. Dai J, Peng L, Fan K, et al: Osteopontin induces angiogenesis through activation of PI3K/AKT and ERK1/2 in endothelial cells. Oncogene 28: 3412-3422, 2009.

39. Iacoangeli A, Lin Y, Morley EJ, et al: BC200 RNA in invasive and preinvasive breast cancer. Carcinogenesis 25: 2125-2133, 2004.

40. Bader AG and Vogt PK: An essential role for protein synthesis in oncogenic cellular transformation. Oncogene 23: 3145-3150, 2004.

41. Kaiser C, Dobrikova EY, Bradrick SS, Shveygert M, Herbert JT and Gromeier M: Activation of cap-independent translation by variant eukaryotic initiation factor $4 \mathrm{G}$ in vivo. RNA 14 2170-2182, 2008

42. Beretta L, Gingras AC, Svitkin YV, Hall MN and Sonenberg N Rapamycin blocks the phosphorylation of 4E-BP1 and inhibits cap-dependent initiation of translation. EMBO J 15: 658-664, 1996.

43. Marcotrigiano J, Gingras AC, Sonenberg N and Burley SK Cap-dependent translation initiation in eukaryotes is regulated by a molecular mimic of elF4G. Mol Cell 3: 707-716, 1999.

44. Herbert TP, Kilhams GR, Batty IH and Proud CG: Distinct signalling pathways mediate insulin and phorbol ester-stimulated eukaryotic initiation factor $4 \mathrm{~F}$ assembly and protein synthesis in HEK 293 cells. J Biol Chem 275: 11249-11256, 2000.
45. Haghighat A, Mader S, Pause A and Sonenberg N: Repression of cap-dependent translation by $4 \mathrm{E}$-binding protein 1: competition with p220 for binding to eukaryotic initiation factor-4E. EMBO J 14: 5701-5709, 1995 .

46. Brunn GJ, Hudson CC, Sekulić A, et al: Phosphorylation of the translational repressor PHAS-I by the mammalian target of rapamycin. Science 277: 99-101, 1997.

47. Chen YJ, Wei YY, Chen HT, et al: Osteopontin increases migration and MMP-9 up-regulation via alphavbeta3 integrin, FAK, ERK, and NF-kappaB-dependent pathway in human chondrosarcoma cells. J Cell Physiol 221: 98-108, 2009.

48. Kumar V, Behera R, Lohite K, Karnik S and Kundu GC: p38 kinase is crucial for osteopontin-induced furin expression that supports cervical cancer progression. Cancer Res 70: 10381-10391, 2010

49. Chang MS, Lee WS, Chen BC, Sheu JR and Lin CH: YC-1-induced cyclooxygenase- 2 expression is mediated by cGMP-dependent activations of Ras, phosphoinositide-3-OH-kinase, Akt, and nuclear factor-kappaB in human pulmonary epithelial cells. Mol Pharmacol 66: 561-571, 2004.

50. Younes M, Lechago LV, Somoano JR, Mosharaf $M$ and Lechago J: Wide expression of the human erythrocyte glucose transporter Glut1 in human cancers. Cancer Res 56: 1164-1167, 1996.

51. Chang SH, Chung YS, Hwang SK, et al: Lentiviral vector-mediated shRNA against AIMP2-DX2 suppresses lung cancer cell growth through blocking glucose uptake. Mol Cells 33: 553-562, 2012.

52. Sommermann TG, Mack HI and Cahir-McFarland E: Autophagy prolongs survival after NF $\mathrm{B}$ inhibition in B-cell lymphomas. Autophagy 8: 265-267, 2012.

53. Park J, Lee HY, Cho MH and Park SB: Development of a cy3-labeled glucose bioprobe and its application in bioimaging and screening for anticancer agents. Angew Chem Int Ed Engl 46: 2018-2022, 2007. 